\title{
Comparison of Auramine Phenol and ZN Staining in Diagnosis of Pulmonary Tuberculosis with GeneXpert MTB/RIF as Gold Standard
}

\author{
Naureen Saeed ${ }^{1}$, Fatima-tuz-zuhra ${ }^{2}$, Nadeem Ikram ${ }^{3}$, Farhana Shaukat ${ }^{4}$, Fareeha Sardar ${ }^{5}$ \\ ${ }^{1}$ Associate Professor, Department of Pathology. Shalimar Medical College Lahore, Pakistan \\ ${ }^{2}$ Assistant professor, Department of Pathology, Rawalpindi Medical University Rawalpindi, Pakistan \\ ${ }^{3}$ Associate Professor, Department of Pathology, Rawalpindi Medical University Rawalpindi, Pakistan \\ ${ }^{4}$ Medical Technologist, District Head Quarter Hospital Rawalpindi, Pakistan \\ ${ }^{5}$ Demonstrator, Rawalpindi Medical University Rawalpindi, Pakistan
}

\begin{abstract}
A B S TRACT
Background: Pakistan faces an immense burden of pulmonary tuberculosis (TB) due to large number of cases and limited resources. Despite the recent advancement in the diagnostic techniques for pulmonary TB, smear microscopy is still a useful technique for the diagnosis of this disease. This study was conducted in order to compare the diagnostic value of Auramine stain with the conventional Ziehl-Neelsen (ZN) stain on the sputum smear for the diagnosis of pulmonary TB, keeping GeneXpert MTB/RIF as the gold standard.

Material and Methods: This cross-sectional study was conducted on 356 suspected patients of pulmonary TB referred to the Pathology laboratory from TB ward and OPD of District Head Quarter (DHQ) teaching hospital Rawalpindi, Pakistan. Sputum specimen were collected and two smears were prepared from each sputum sample on which Auramine phenol and Ziehl-Neelsen staining were carried out as per WHO recommendations. All these samples were further tested using Gene Xpert MTB/RIF technique. The sensitivity, specificity, Positive predictive value (PPV) and Negative predictive values (NPV) of ZN and Auramine stain were calculated and compared with GeneXpert MTB/RIF technique.

Results: Out of the total 356 samples, 64 (18\%) were positive and 291 (82\%) were negative by GeneXpert which was taken as the gold standard. On comparison with GeneXpert, percentage of true positive was greater in case of Auramine than ZN stained samples (16.29\% versus $12.92 \%$ ), while the percentage of false positive was same for both staining techniques (0.28\%). There were lesser false negative cases observed in samples stained by Auramine as compared to the ones stained by ZN (1.68\% versus $5.05 \%)$. The sensitivity, specificity, positive predictive value and negative predictive values were $97.87 \%, 94.17 \%, 71.88$ and $99.66 \%$, respectively for the ZN staining and $98.31 \%, 97.98 \%, 90.63 \%$ and $99.66 \%$ respectively, for the Auramine phenol staining.

Conclusions: Smear microscopy using Auramine phenol stain is a useful technique for the diagnosis of pulmonary TB. The Auramine phenol staining with fluorescent microscopy is found to be superior to ZN staining because of higher sensitivity and specificity.

Key words: Auramine phenol, Fluorescence microscopy, GeneXpert, Mycobacterium tuberculosis, Ziehl-Neelsen

\begin{tabular}{lll} 
Authors' Contribution: & Correspondence: & Article info: \\
1,2Conception; Literature research; & Fatima-tuz-zuhra & Received: October 15, 2019 \\
manuscript design and drafting; ${ }^{3,4}$ Critical & Email:fatizuhra2@gmail.com & Accepted: September 15, 2020 \\
$\begin{array}{l}\text { analysis and manuscript review; Data } \\
\text { analysis; Manuscript Editing. }\end{array}$ & & \\
\hline
\end{tabular}
\end{abstract}

Cite this article. Saeed N, Zuhra F, Ikram N, Shaukat F, Sardar F. Comparison of Auramine Phenol and ZN Staining in Diagnosis of Pulmonary Tuberculosis with GeneXpert MTB/RIF as Gold Standard. J Islamabad Med Dental Coll. 2020; 9(3): 190-194. Doi:

Funding Source: Nil Conflict of Interest: Nil 10.35787/jimdc.v9i3.435 


\section{Introduction}

Pulmonary TB, caused by Mycobacterium tuberculosis (MTB) is a contagious disease that involves the lungs, and may spread to other organs. ${ }^{1}$ Tuberculosis is a worldwide health problem and one of the leading causes of death even in the present era, despite the fact that the disease is preventable and curable. In the year 1993, World Health Organization (WHO) declared TB as a global emergency. In spite of the efforts made for its control and treatment, pulmonary TB is still a worldwide health problem, especially in the developing countries. In the year 2012, WHO estimated 8.6 million people infected with TB and 1.3 million deaths from the disease. Moreover, a large number of people developed multidrugresistant tuberculosis (MDR-TB), which remains one of the biggest medical challenge to date. ${ }^{1}$

Pulmonary tuberculosis is an important health problem in Pakistan, with an estimated 510,000 new cases emerging each year and approximately 15,000 developing drug resistant TB. ${ }^{2}$ Each active, sputum positive case of pulmonary tuberculosis can infect 10-15 people in a year. However, for effective management and disease control, an early and accurate diagnosis of TB and MDR-TB is crucial. ${ }^{3}$

Diagnosis of pulmonary TB is usually based on positive family history, presence of classical clinical features (weight loss, cough, hemoptysis), positive findings on $\mathrm{X}$-rays and Purified Protein Derivative (PPD) results. ${ }^{4}$ Nevertheless, the final diagnosis is based on laboratory results of sputum examination. ${ }^{5}$ For decades, direct microscopy of sputum smear of susceptible cases has been widely used for the diagnosis of TB. Ziehl-Neelsen and Auramine phenol are the two most commonly used stains for the detection of acid-fast bacilli (AFB) in the sputum of patients with pulmonary TB. ZN staining of sputum smear is in use since 1882 and is an economical method of diagnosis of pulmonary TB. ${ }^{5}$ Fluorescence staining was introduced in 1978 for sputum examination. ${ }^{6}$

In 2011, WHO introduced GeneXpert MTB/RIF assay for the detection of Mycobacterium tuberculosis. It is an automated real time PCR (RT-PCR) which utilizes a DNA-PCR technique. ${ }^{7}$ It also has provision of nucleic acid extraction, amplification, an advantage of bacterial lysis and minimum biosafety requirements. ${ }^{8}$ Since its introduction GeneXpert has revolutionized the detection of Mycobacterium TB in all kind of difficult to diagnose cases. ${ }^{9}$ In this study we compared the diagnostic accuracy of the two conventional sputum staining methods with GeneXpert as gold standard in diagnosing pulmonary TB.

\section{Material and Methods}

This cross-sectional study was carried out at the Department of Pathology, District Head Quarter hospital Rawalpindi, Pakistan for a duration of 6 months, from $1^{\text {st }}$ January to $30^{\text {th }}$ June 2019 . A total of 356 sputum samples were included in the study. All the samples were taken from patients with a strong clinical suspicion of pulmonary TB referred from OPD and TB ward of DHQ hospital Rawalpindi. Strong clinical suspicion was based on cough, weight loss, and hemoptysis, positive PPD results and positive chest X-ray findings. Samples with insufficient volume or contaminated with food particles were excluded. The study was approved by the Institutional Review Board (IRB) of DHQ hospital, Rawalpindi.

Each sputum sample was divided into three parts; one used for ZN smear, second for Auramine phenol smear and the third part for GeneXpert testing.

The sputum smear prepared for ZN staining was flooded with carbol fuchsin and the slide was heated 
for 3-5 minutes and then rinsed again with water, decolorized and dried.

Microscopic examination was done at $100 x$ for at least 5 minutes. Each staining batch included a positive and negative slide for quality control. Interpretation of $\mathrm{ZN}$ staining was done as per Revised National Tuberculosis Program (RNTCP) guidelines. Slides with red stained rods against blue background were labeled positive, whereas those without any rods seen on microscopy were labeled negative.

For Auramine phenol staining, the smear was prepared, dried and $0.1 \%$ Auramine phenol was poured on the slide for 20 minutes, washed with water, decolorized with acid-alcohol and counterstained with methylene blue. The stained slides were examined under LED fluorescent microscope at 20x for at least 3 minutes by scanning the slide from one side to the other. This was equivalent to one length or 100 high-power fields. The smears were graded according to $\mathrm{WHO}$ recommendations as $3+, 2+, 1+$, doubtful and negative.

For GeneXpert $0.5 \mathrm{ml}$ of sputum sample was transferred to a sterile tube, $1.5 \mathrm{ml}$ sample reagent was added and allowed to stand at room temperature for 15 minutes. Then $2 \mathrm{ml}$ of the mixture was transferred to the test cartridge and placed in the GeneXpert device. The results of the tests were noted and interpretation was done after 2 hours.

Statistical analysis was done using SPSS software version 22 (Chicago IL). The sensitivity, specificity, Positive Predictive values (PPV) and Negative Predictive values (NPV), for ZN and Auramine phenol staining techniques were calculated.

\section{Results}

Of the total 356 samples, 64 (18\%) were positive and 291 (82\%) were negative by GeneXpert, which was taken as the gold standard. On comparison with
GeneXpert, percentage of true positive sampleswas greater in case of Auramine-stained than $\mathrm{ZN}$-stained samples (16.29\% versus $12.92 \%)$, while the percentage of false positive was same for both staining techniques (0.28\%). Similarly, few false negative cases were observed in samples stained by Auramine as compared to the ones stained by ZN (1.68\% versus $5.05 \%$ ) (Table I).

Table I: Results of Ziehl-Neelsen and Auramine phenol staining methods compared with GeneXpert $(n=356)$

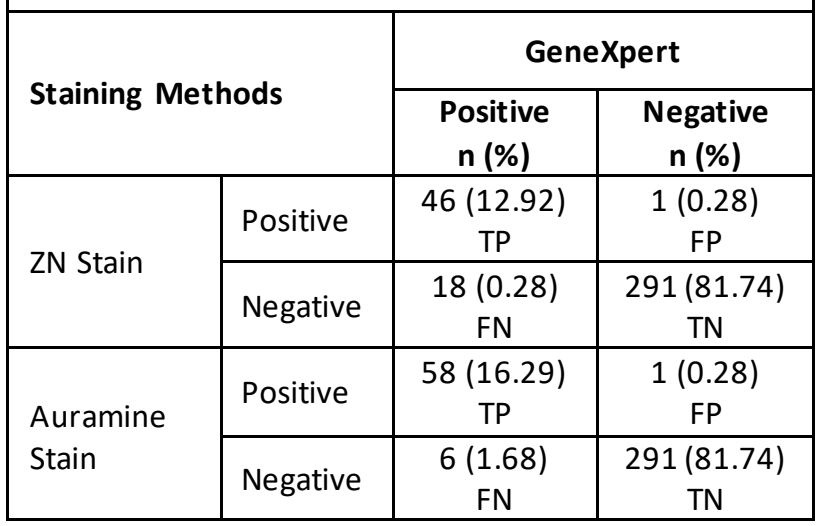

TP-True positive; FP-False positive; TN-True negative; FNFalse negative

Auramine staining was found to be more sensitive and specific than Ziehl-Neelsen stain in detecting Mycobacterium tuberculosis ( $98.31 \%$ versus $97.87 \%$ and $97.98 \%$ versus $94.17 \%$ ). It also has much higher positive predictive value $(90.63 \%$ versus $71.88 \%)$ than ZN staining (Table II).

\begin{tabular}{|c|c|c|}
\hline \multicolumn{3}{|c|}{$\begin{array}{l}\text { Table II: Sensitivity, specificity and predictive values } \\
\text { for Ziehl-Neelsen and Auramine phenol staining } \\
\text { methods }(n=356)\end{array}$} \\
\hline & \multicolumn{2}{|c|}{ Staining Methods } \\
\hline & ZN Stain & Auramine Stain \\
\hline Sensitivity (\%) & 97.87 & 98.31 \\
\hline Specificity (\%) & 94.17 & 97.98 \\
\hline PPV (\%) & 71.88 & 90.63 \\
\hline NPV (\%) & 99.66 & 99.66 \\
\hline
\end{tabular}

PPV-Positive predictive value; NPV-Negative predictive value 


\section{Discussion}

In developing countries like Pakistan, microscopy of sputum smear for the detection of AFB is still used for the diagnosis of pulmonary TB since it is a reliable, economical and rapid method of detection. ${ }^{10}$ In the present study two staining techniques, ZN and Auramine phenol were compared with GeneXpert as a gold standard. The results were in favor of Auramine phenol and showed that it is better in terms of sensitivity, specificity and positive predictive value than ZN staining technique. These results are in-line with the findings of many other studies. Chhina et al. found highervalues for sensitivity, specificity, PPV and NPV of Auramine staining $(100 \%, 99.8 \%, 99.5 \%$ and $100 \%$ respectively), as well as ZN staining (85.58\%, 100\%, $100 \%$ and $96.3 \%$ respectively). ${ }^{11}$ Similarly, another study by Hooja et al. showed $55.55 \%$ and $71.85 \%$ sensitivity values for $\mathrm{ZN}$ and Auramine stains respectively. The sensitivity of AFB smear was further increased by $27.41 \%$ when both methods were combined. ${ }^{12}$

Habeenzu et al. found 152 sputum specimens positive with Auramine staining and 66 with ZN staining method. ${ }^{13}$ Ulukanligil et al. also found higher sensitivity of Auramine stain (85.3\%) as compared to ZN stain (76.6\%) in their study. ${ }^{10}$

Our findings showed that both $\mathrm{ZN}$ and Auramine staining techniques can be effectively utilized for the diagnosis of pulmonary TB. However, Auramine stain is more sensitive in detecting lesser number of bacilli in smears which may remain undetected with ZN staining. ${ }^{10}$ These findings are corroborating the results of studies by Ulukanligill et al. and Murray et al. ${ }^{10,14}$ Murray and colleagues compared the sensitivity of Auramine phenol and ZN staining at all of the five stages of sample preparation and declared Auramine phenol stain to be more sensitive and reliable than ZN staining in detection of acid-fast bacilli (AFB). ${ }^{14}$ Lack of requirement of flame for slide fixation and examination of larger slide area at lower magnification and in lesser time, are the other advantages of Auramine staining over $\mathrm{ZN}$ staining. ${ }^{6}$

We acknowledge that the results of these staining techniques are influenced by multiple factors like skill at staining, quality of stains, expertise of examining slides and adherence to the standard guidelines. However, we believe that despite the availability of advanced techniques such as GeneXpert, there is still an advantage of performing sputum smear microscopy. This is because a patient established TB-positive by both methods is likely to be more infectious than the one who is smearnegative but positive on GeneXpert. ${ }^{15}$ The costeffectiveness also makes it a preferable method for health care settings facing budget constraints and having large number of samples for pulmonary TB screening.

\section{Conclusion}

Smear microscopy using Auramine phenol stain is a useful technique for the diagnosis of pulmonary TB and is superior to $\mathrm{ZN}$ staining in terms of sensitivity and specificity.

\section{References}

1. Zumla A, George A, Sharma V, Herbert N, Baroness Masham of Ilton. WHO's 2013 global report on tuberculosis: successes, threats, and opportunities. Lancet 2013; 382(9907): 1765-7. Doi: 10.1016/S0140-6736(13)62078-4.

2. World Health Organization. Global tuberculosis report 2018. World Health Organization. 2018. http://www.who.int/iris/handle/10665/274453

3. Agrawal M, Bajaj A, Bhatia V, Dutt S. Comparative Study of GeneXpert with ZN Stain and Culture in Samples of Suspected Pulmonary Tuberculosis. J Clin Diagn Res. 2016; 10(5): DC09-12. Doi: 10.7860/JCDR/2016/18837.7755.

4. Waard JH, Robledo J. Conventional diagnostic methods. In: Palomino JC, Leão SC, Ritacco V (eds). 
Tuberculosis (www.TuberculosisTextbook.com) 2007; 12: 401-24.

5. Centers for Disease Control and Prevention (CDC). Updated guidelines for the use of nucleic acid amplification tests in the diagnosis of tuberculosis. MMWR Morb Mortal Wkly Rep. 2009; 58: 7-10.

6. Balakrishna J, Shahapur PR, Chakradhar P, Hussain Saheb S. Comparative Study of Different Staining Techniques - Ziehlneelsen Stain, Gabbet's Stain, Fluorochrome Stain for Detecting of Mycobacterium Tuberculosis in the Sputum. J Pharm Sci \& Res. 2013; 5(4): 89-92.

7. Li S1, Liu B1, Peng M1, Chen M1, Yin W1, Tang $H$. Diagnostic accuracy of Xpert MTB/RIF for tuberculosis detection in different regions with different endemic burden: A systematic review and meta-analysis. PLoS One. 2017; 12(7): e0180725. Doi: 10.1371/journal.pone.0180725

8. Saeed M, Iram S, Hussain S, Ahmed A, Akbar M, Aslam M. GeneXpert: A new tool for the rapid detection of rifampicin resistance in mycobacterium tuberculosis. J Pak Med Assoc. 2017; 67(2): 270-74.

9. Rakotoarivelo R, Ambrosioni J, Rasolofo V, Raberahona M, Rakotosamimanana N, Andrianasolo R. Evaluation of the Xpert MTB/RIF assay for the diagnosis of smear-negative pulmonary and extrapulmonary tuberculosis in Madagascar. Int J Infect Dis. 2018; 69: 20-25. Doi: 10.1016/ j.ijid.2018.01.017
10. Ulukanligil M, Aslan G, Tasci S. A comparative study on the different staining methods and number of specimens for the detection of acid-fast bacilli. Mem Inst Oswaldo Cruz. 2000; 95: 855-58.

11. Chhina D, Gupta R, Chawla A. Comparison of Ziehl Neelsen (ZN) and Auramine Phenol (AP) Staining Method to Detect Acid-Fast Bacilli in Sputum Smear. IJRHS. 2018; 6(1): 1-5.

12. Hooja S, Pal N, Malhotra B, Goyal S, Kumar V, Vyas L. Comparison of Ziehl Neelsen \& Auramine O staining methods on direct and concentrated smears in clinical specimens. Indian J Tuberc. 2011; 58(2): 72-6. PMID: 21644393

13. Habeenzu C, Lubasi D, Fleming AF. Improved sensitivity of direct microscopy for detection of acidfast bacilli in sputum in developing countries. Trans $R$ Soc Trop Med Hyg. 1998; 92(4): 415-16. Doi: 10.1016/s0035-9203(98)91071-9

14. Murray SJ, Barrett A, Magee JG, Freeman R. Optimization of acid-fast smears for the direct detection of mycobacteria in clinical samples. J Clin Pathol. 2003; 56(8):613-15. Doi: 10.1136/jcp. 56.8 .613

15. Rachow A, Zumla A, Heinrich N, Rojas-Ponce G, Mtafya B, Reither K. Rapid and accurate detection of Mycobacterium tuberculosis in sputum samples by Cepheid Xpert MTB/RIF assay -- a clinical validation study. PLoS One. 2011; 6(6): e20458. Doi: 10.1371/journal.pone. 0020458. 